Portrait comparatif des activités de développement professionnel privilégiées en enseignement en contextes préscolaire, primaire et secondaire Portrait of comparative activities of preferred professional development in preschool, primary and secondary contexts Retrato comparativo de las actividades de desarrollo profesional privilegiadas en la enseñanza en contextos de preescolar, primaria y secundaria

Nathalie Murray

Volume 40, numéro 1, 2014

Texte reçu le : 28 novembre 2013, version finale reçue le : 19 août 2014, accepté le : 19 août 2014

URI : https://id.erudit.org/iderudit/1027625ar

DOI : https://doi.org/10.7202/1027625ar

Aller au sommaire du numéro

Éditeur(s)

Revue des sciences de l'éducation

ISSN

0318-479X (imprimé)

1705-0065 (numérique)

Découvrir la revue

Citer cet article

Murray, N. (2014). Portrait comparatif des activités de développement professionnel privilégiées en enseignement en contextes préscolaire, primaire et secondaire. Revue des sciences de l'éducation, 40(1), 107-128.

https://doi.org/10.7202/1027625ar
Résumé de l'article

Cet article présente une recherche quantitative qui a permis de dresser un portrait comparatif des activités privilégiées par les enseignants en exercice, en contextes préscolaire, primaire et secondaire pour le développement des compétences professionnelles, en lien avec le profil du référentiel de la formation en enseignement au Québec. L'utilité perçue de ces activités a été mesurée. Cette recherche fait appel à la technique d'enquête, et nous avons élaboré un questionnaire dans ce but. Les résultats indiquent que les activités proposées sont utilisées à des fréquences variables selon le genre, le nombre d'années d'expérience, l'ordre et le champ d'enseignement. Néanmoins, le développement de certaines compétences est délaissé, ce qui soulève de nombreuses questions. Nous vous exposons la démarche et quelques résultats. 


\title{
Portrait comparatif des activités de développement professionnel privilégiées en enseignement en contextes préscolaire, primaire et secondaire
}

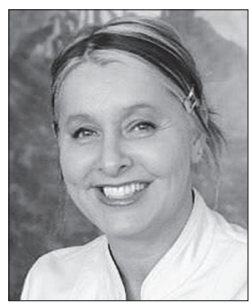

Nathalie Murray

Conseillère pédagogique

Université du Québec à Trois-Rivières

\begin{abstract}
RÉSUMÉ - Cet article présente une recherche quantitative qui a permis de dresser un portrait comparatif des activités privilégiées par les enseignants en exercice, en contextes préscolaire, primaire et secondaire pour le développement des compétences professionnelles, en lien avec le profil du référentiel de la formation en enseignement au Québec. L'utilité perçue de ces activités a été mesurée. Cette recherche fait appel à la technique d'enquête, et nous avons élaboré un questionnaire dans ce but. Les résultats indiquent que les activités proposées sont utilisées à des fréquences variables selon le genre, le nombre d'années d'expérience, l'ordre et le champ d'enseignement. Néanmoins, le développement de certaines compétences est délaissé, ce qui soulève de nombreuses questions. Nous vous exposons la démarche et quelques résultats.
\end{abstract}

MOTS CLÉS • activités, compétences, développement professionnel.

\section{Introduction et problématique}

Cette recherche quantitative s'est inspirée de divers contextes ayant orienté le développement professionnel en enseignement au Québec: celui du renouveau pédagogique et celui de la professionnalisation de l'enseignement. Ils ont également influencé la mission éducative québécoise et plus particulièrement le rôle de l'enseignant. Il en ressort que l'enseignant est l'acteur principal en éducation et que ses interventions pédagogiques agissent directement sur la qualité des apprentissages de l'élève. Par conséquent, depuis plusieurs années, les établissements scolaires et le ministère de l'Éducation, du Loisir et du Sport (MELS) ont mis en place des activités de formation continue en période de développement 
professionnel afin de permettre à l'enseignant d'améliorer ses interventions pédagogiques.

Cette recherche, faite dans le cadre d'une thèse, dresse un portrait des activités pour mieux cerner le développement des compétences professionnelles, en examinant les activités privilégiées par les enseignants et leur utilité perçue dans l'enseignement au quotidien. Diverses variables sont prises en compte: l'ordre d'enseignement, le nombre d'années d'expérience, le genre, le champ d'enseignement et les compétences visées. La population à l'étude est formée des enseignants de la région du Saguenay-Lac-Saint-Jean, d'abord choisie en fonction de sa représentativité.

Dans cet article, nous exposons le contexte, la problématique, les questions et les objectifs de la recherche. Puis, une brève description des concepts abordés, de la méthodologie employée ainsi que quelques résultats sont présentés avant de conclure.

\subsection{Principal acteur en éducation}

Selon de nombreuses recherches, l'enseignant est un acteur primordial en éducation et il doit posséder un ensemble de compétences pour exercer ses fonctions (Craft, 2000; Girard, McLean et Morissette, 1992; Presseau et Frenay, 2004; Tardif, 2006). Allal (2002), De Bock (2000) et Holh (1993) affirment que les enseignants ont une influence sur la qualité des apprentissages des élèves, bien plus que les autres intervenants qui les entourent. Des rapports américains, canadiens et européens (Learned et Bagley, 1988; Lesourne, 1988; Organisation de coopération et de développement économiques-OCDE, 1998) montrent que ce sont eux qui font la différence, principalement dans l'apprentissage et la réussite des élèves. Par ailleurs, on dénombre, de nos jours, nettement plus de recherches qui aident à mieux comprendre la nature et la pertinence des pratiques enseignantes visant à favoriser l'apprentissage des élèves (Brassard, 2008; Bru, 2004; Gauthier et Mellouki, 2003; Massot et Feisthammel, 1997).

\subsection{Développement des compétences professionnelles}

Le développement des compétences professionnelles vise l'amélioration de l'intervention pédagogique pour contribuer à la progression de l'apprentissage scolaire des élèves (Bouteiller, 2009; Gohier, Anadón, Bouchard, Charbonneau, et Chevrier 1999; Paquay, 2000a). Des auteurs associent le développement des compétences au développement professionnel, puisqu'elles doivent être mises à jour de façon continue, afin de tenir compte de l'évolution de la profession et de la société, de même que du développement de la recherche en éducation (Altet, 2001; Anderson, 1986; Flück, 2001; Kalika, 1998; Perrenoud, 1999; Presseau, Martineau et Miron, 2002).

En ce sens, le ministère de l'Éducation du Québec (1999) a investi des sommes importantes dans le soutien au développement professionnel (Gauthier et Mellouki, 2003). Il sollicite la responsabilité des enseignants face à leur propre 
formation continue, afin qu'ils s'investissent dans un processus de développement professionnel en pratiquant diverses activités, et ce, malgré les conditions dans lesquelles elles sont accessibles ou offertes, comme l'horaire, le lieu et le temps (Martineau et Gauthier, 2000). D’ailleurs, la perception de l'utilité des activités est fondamentale pour s'engager dans un processus de développement professionnel en formation continue (Saint-Pierre et Lison, 2012).

Cependant, le type de soutien offert aux enseignants est souvent occasionnel et prend la forme d'un perfectionnement uniforme et obligatoire. Ce type de soutien laisse donc davantage de place à des démarches de prise en charge individuelle (Brassard, 2008). Conséquement, la lourdeur de la tâche d'enseignement due aux changements socioéconomiques et politiques ainsi que les remises en question individuelles incitent les enseignants à demeurer prudents face à leurs démarches de développement professionnel (Carnus, Garcia-Debanc et Terrisse, 2008; Portelance, Mukamurera, Martineau et Gervais, 2008; Théberge, Bourrassa, Lauzon et Huard-Wart, 1997).

\subsection{Situation actuelle}

Au Québec, depuis plus de dix ans, les enseignants en exercice ont eu accès à des offres d'activités de développement professionnel prescrites par le ministère de l'Éducation ou offertes par leurs institutions respectives, et ce, dans toutes les régions. Par contre, il semble bien que les buts ciblés par ces offres n'aient pas eu les résultats escomptés puisqu'elles se heurtent à des obstacles: résistance des enseignants, lourdeur de la tâche, etc. (Prud'homme, 2007).

Étant donné cette réalité, cette recherche vise à apporter un éclairage nouveau sur les activités privilégiées par les enseignants afin de développer leurs compétences professionnelles, de même que sur l'utilité perçue de celles-ci dans l'enseignement au quotidien, et ce, selon diverses variables. Cette recherche répond aux questions suivantes:

Quelles sont les activités de développement professionnel, en lien avec les compétences du référentiel de la formation en enseignement, privilégiées par les enseignants en exercice, en contextes préscolaire, primaire et secondaire et quelles sont les différences entre ces activités en fonction de l'ordre d'enseignement, du nombre d'années d'expérience, du sexe et du champ d'enseignement?

Quelle est l'utilité, perçue par les enseignants, des activités de développement professionnel dans leur enseignement au quotidien et comment cette utilité perçue varie-t-elle en fonction de l'ordre d'enseignement, du nombre d'années d'expérience, du genre, du champ d'enseignement et des compétences visées?

L'objectif général de cette recherche est donc de dresser un portrait comparatif des activités de développement professionnel, en lien avec les compétences du référentiel de la formation en enseignement, privilégiées par les enseignants en exercice, au secteur éducatif pour les jeunes, en contextes préscolaire, primaire 
et secondaire. Les objectifs spécifiques sont de 1) répertorier et analyser les activités de développement professionnel privilégiées par les enseignants en exercice, 2) comparer ces activités de développement professionnel selon diverses variables, telles que le genre, le nombre d'années d'expérience, l'ordre et le champ d'enseignement, 3) mesurer l'utilité perçue par les enseignants de ces activités dans le cadre de leur enseignement au quotidien, et enfin, 4) comparer cette utilité perçue selon diverses variables, telles que le genre, le nombre d'années d'expérience, l'ordre et le champ d'enseignement.

\subsection{Pertinence de la recherche}

Cette recherche vise à contribuer à l'apport de connaissances, mais également à offrir un cadre de réflexion en vue du renouvellement ou de la bonification d'activités de développement professionnel en lien avec les douze compétences professionnelles du Référentiel de la formation en enseignement (ministère de l'Éducation du Québec, 2001). De plus, le portrait dégagé permettra de comprendre l'utilité perçue des pratiques déployées, d'autant plus qu'il aura été nuancé en fonction de diverses variables.

\section{Cadre conceptuel}

Deux concepts sont retenus, celui de compétence et celui de développement professionnel. Une analyse des concepts abordés permet de dégager une compréhension commune de ceux-ci et de spécifier certains choix méthodologiques.

\subsection{Concept de compétence}

Cette recherche porte sur le développement des compétences professionnelles et sur l'apport du profil des compétences du Référentiel de la formation en enseignement au Québec comme modèle de base vers lequel doivent converger toutes les activités de développement professionnel. Dans ce référentiel (ministère de l'Éducation du Québec, 2001), douze compétences sont définies et dressent clairement le profil de compétences que doit développer un enseignant, tout au long de sa carrière. Cependant, les définitions de compétence sont nombreuses et découlent de perspectives variées. En effet, l'analyse du concept a été effectuée par plusieurs auteurs, notamment en éducation (Jonnaert, 2002; Le Boterf, 2001; Martineau, 2006), en sociologie (Anderson, 1986; Levy-Leboyer, 1996) et en psychologie (Perrenoud, 2001; Tardif, 2006).

Pour baliser la signification de ce concept en lien avec cette recherche, à la suite de la recension et inspirée de ces perspectives, nous définissons la compétence comme la capacité qu'a une personne à exploiter efficacement ses ressources personnelles, à faire preuve de jugement et de décision dans un processus dynamique et évolutif lié à un environnement contextualisé. La compétence n'est pas statique et stable; elle évolue, car elle est dynamique. C'est en ce sens que des auteurs comme Altet (2001), Anderson (1986), Flück (2001), Kalika (1998), 
Perrenoud (1999), de même que Presseau et ses collaborateurs (2002) ont associé le développement des compétences au développement professionnel.

\subsection{Concept de développement professionnel}

Deux perspectives principales ressortent dans les façons de définir le développement professionnel. D’une part, il est conçu comme un continuum qui commence dès la période de la formation initiale (Altet, 2002; Paquay, 2000b; Parent, Corriveau, Savoie-Zajc, Dolbec, Cartier, Toussaint, Laurin, et Bonneau, 1999; Pinte, 2004; Théberge et al., 1997. D’autre part, il peut être vu comme un processus de perfectionnement qui fait suite à la formation initiale et qui requiert la mise en place de stratégies de formation continue (Anadón, 1999; Hargreaves, 1995; Hendricson et Kleffner, 1998). Pour la présente recherche, la perspective de processus a été retenue et les participants ont été ciblés en ce sens.

\subsubsection{Les activités de développement professionnel}

Le développement professionnel requiert la pratique d'activités variées telles qu'assister à un colloque ou à des conférences, suivre un cours, faire des lectures, se former sur le tas ou par essais et erreurs, cumuler des expériences et essayer de nouvelles techniques (Batal, 1997; Bélair, 2001; Glaser, 1987; Wittorski, 2000). À cette liste, Schön (1994) a ajouté la réflexion, alors que Batal (1997) et Wittorski (2004) parlent d'analyse des pratiques et d'autocritique. Pour d'autres, la concertation, la collaboration, les stages, le tutorat, la formation par des collègues ou des professionnels, la consultation de ceux-ci ainsi que la participation à un projet de recherche peuvent faire partie des activités de développement professionnel (Altet, 1996; Bélair, 2001 ; Le Boterf, 2001 ; Parent et al., 1999).

De plus, des auteurs ont tenté de classifier ces activités (Guillemette, 2006; Wittorski, 2007). Par exemple, Wittorski (2007) a proposé six voies de développement professionnel qui représentent la logique du développement professionnel. De son côté Guillemette (2006) a analysé et dégagé les voies du développement professionnel utilisées par les enseignants. Il a identifié quatre grandes voies de développement professionnel qui se présentent en simultanéité dans les expériences concrètes. Le tableau 1 suivant détaille les voies répertoriées par ces deux auteurs (Guillemette, 2006; Wittorski, 2007).

Cependant, la catégorisation de tous les types d'activités dans ces voies est difficile si, préalablement, une analyse précise de ce qui est investi n'est pas faite. Dans cette recherche, d'un point de vue praxéologique, pour les besoins de la collecte et de l'analyse de données, une nouvelle typologie a été proposée en lien avec les écrits recensés. Celle-ci inclut la logique de la réflexion dans toutes les voies qu'elle considère, puisqu'elle est partie intégrante du processus de développement professionnel. Cette considération est liée aux études des différents auteurs qui ont dégagé la pratique réflexive comme un élément sans équivoque qui contribue au développement professionnel (Batal, 1997; Schön, 1994; Wittorski, 
Tableau 1

Voies du développement professionnel

\begin{tabular}{ll}
\hline \multicolumn{1}{c}{ Wittorski (2007) } & \multicolumn{1}{c}{ Guillemette (2006) } \\
\hline Logique de la réflexion sur l'action & L'exploitation des ressources internes \\
Logique de la réflexion pour l'action & L'exploitation des ressources externes \\
Logique de la traduction culturelle par rapport à l'action & La dimension collégiale \\
Logique de l'action & La pratique réflexive \\
Logique de la réflexion et de l'action & \\
Logique de l'intégration/assimilation & \\
\hline
\end{tabular}

2004). L'utilisation de cette typologie a facilité la classification des activités. Dans celle-ci, les voies présentées se regroupent en trois grandes catégories: la dimension interne, la dimension externe et la dimension combinée.

\subsubsection{Dimension interne}

La catégorie de la dimension interne est en lien avec la perspective de plusieurs auteurs, selon lesquels le développement professionnel présente une dimension personnelle et individuelle qui demande de mobiliser et de transférer l'ensemble des ressources internes de la personne (les connaissances et les compétences acquises) pour formaliser ses propres pratiques et dégager des modèles à reproduire (Le Boterf, 2008; Parent et al., 1999; Presseau et al., 2002; Toupin, 1995; Wittorski, 2000). Pour ces mêmes auteurs, la dimension interne inclut la réflexion nécessitant l'ajustement habituel dans le processus de l'action, ce qui transforme l'acte d'enseigner en expérience formalisée. Elle est à caractère rétrospectif ou prospectif. Elle consiste à faire l'analyse de ses pratiques ainsi qu'une autocritique. Cette dimension fait également référence à la période d'autoformation où il s'agit, par exemple, d'essayer de nouvelles stratégies (tableau 2).

\subsubsection{Dimension externe}

Selon plusieurs auteurs (Hargreaves, 1995; Lebuis, Bednarz et Desgagné, 1995; Paquay, 2000b; Savoie-Zajc et Dionne, 2001), le développement professionnel des enseignants peut se faire par la mobilisation de ressources externes, qui peut être individuelle ou collective, mais dans un but de résolution de problèmes. Du côté individuel, la personne peut participer à des colloques, consulter des documents ou des pairs, par exemple. Enfin, en tenant compte de la perspective de collectivité, les activités sont centrées sur le partage d'expertises (tableau 2).

\subsubsection{Dimension combinée}

D’autres auteurs mentionnent que les activités de développement professionnel peuvent mobiliser simultanément des ressources internes et externes de la personne (Clément et Vanderberghe, 2000; Cochran-Smith et Lytle, 1999; Courcy, 2002; Leclerc, 2012; Lieberman et McLaughlin, 2000; Paquette, 2002). Cette 
dimension combinée peut se traduire par diverses activités: appartenance à un groupe de codéveloppement ou accompagnement d'un stagiaire, entre autres. Cette dimension peut également inclure la lecture, un cours suivi ou un engagement personnel dans un processus de recherche. Le tableau 2 regroupe toutes ces activités recensées ayant servi à l'élaboration du questionnaire en fonction de la typologie des trois dimensions.

\section{Tableau 2}

Activités de développement professionnel selon les dimensions

\begin{tabular}{|c|c|c|}
\hline $\begin{array}{l}\text { Dimension interne } \\
\text { (DI) }\end{array}$ & $\begin{array}{l}\text { Dimension externe } \\
\text { (DE) }\end{array}$ & $\begin{array}{l}\text { Dimension combinée } \\
\text { (DC) }\end{array}$ \\
\hline \multirow{4}{*}{$\begin{array}{l}\text { Appliquer de nouvelles } \\
\text { techniques ou stratégies } \\
\text { d'enseignement }\end{array}$} & \multirow{4}{*}{$\begin{array}{l}\text { Assister à des colloques } \\
\text { ou à des conférences } \\
\text { Collaborer à des projets avec } \\
\text { l'équipe enseignante }\end{array}$} & \multirow{4}{*}{$\begin{array}{l}\text { Suivre un cours } \\
\text { Se concerter entre collègues } \\
\text { ou intervenants } \\
\text { Accompagner un stagiaire }\end{array}$} \\
\hline & & \\
\hline & & \\
\hline & & \\
\hline \multirow[t]{6}{*}{$\begin{array}{l}\text { S'autocritiquer ou analyser ses } \\
\text { pratiques }\end{array}$} & \multirow{6}{*}{$\begin{array}{l}\text { Consulter des documents ou } \\
\text { ouvrages de référence } \\
\text { Consulter des professionnels } \\
\text { Participer à une formation } \\
\text { donnée par les pairs ou par les } \\
\text { professionnels }\end{array}$} & $\begin{array}{l}\text { Participer à un programme de } \\
\text { tutorat ou de mentorat }\end{array}$ \\
\hline & & \multirow{3}{*}{$\begin{array}{l}\text { Faire des lectures sur des sujets } \\
\text { d'intérêt professionnel } \\
\text { Participer à un groupe } \\
\text { d'analyse, à une communauté } \\
\text { professionnelle ou à un groupe } \\
\text { de codéveloppement }\end{array}$} \\
\hline & & \\
\hline & & \\
\hline & & $\begin{array}{l}\text { Participer à des recherches } \\
\text { collaboratives ou action menées } \\
\text { par un chercheur externe }\end{array}$ \\
\hline & & $\begin{array}{l}\text { S'engager personnellement } \\
\text { dans un processus de recherche }\end{array}$ \\
\hline
\end{tabular}

\section{Méthodologie}

Une recension et une analyse des différentes recherches portant sur l'objet d'étude, le développement des compétences professionnelles, ont été réalisées. La majorité de ces recherches utilise l'approche qualitative avec ses différentes techniques (Anderson, 1986; Becher, 1999; Campbell, Cignetti, Melenyzer, Nettles et Wyman 1997; Day, 1999; Fourez, 1999; Guskey, 2000; Hargreaves, 1995; Hendricson et Kleffner, 1998; Holborn, 1992; Lafortune, Deaudelin et Deslandes, 2001; Van Der Heijden et Barbier, 1999). Ces approches s'appuient sur un petit nombre de participants, permettent de mieux comprendre le phénomène du développement professionnel et d'analyser les pratiques, mais sans nuancer les activités utilisées en fonction de diverses variables ni mesurer la perception de leur utilité. D’autres recherches qualitatives font émerger différentes catégories d'activités de développement professionnel ou encore ciblent les phases liées à ce développement (Guillemette, 2006; Huberman, 1989; Vonk, 1988). Enfin, des recherches théo- 
riques recensent diverses théories existantes et permettent une réflexion sur la validité de celles-ci (Altet et Fabre, 1994; Durand, 1996; Mukamurera, Bourque et Gingras, 2008; Orange, 2006; Wittorski, 2000). Peu de recherches quantitatives ont été recensées sur le développement professionnel. Par exemple, celle de Daele et Charlier (2004) consistait en une étude exploratoire, qui visait à recenser des besoins liés aux communautés de développement professionnel, tandis que celle de Buhot et Cosnefroy (2011) avait été menée dans le but de connaître la perception du développement professionnel des enseignants en formation initiale et en première année d'insertion.

Avec la présente recherche, nous avons voulu dresser un portrait comparatif des activités privilégiées chez les enseignants pour développer leurs compétences professionnelles. Ainsi, une large population a été ciblée pour permettre une comparaison et une analyse selon diverses variables. Dans cette perspective, l'approche quantitative, associée à des techniques d'enquête, a été privilégiée puisqu'elle répond efficacement aux objectifs visés, qu'elle permet d'avoir une large population à l'étude, en plus d'être en lien avec les choix méthodologiques, procéduraux et techniques ainsi qu'avec les outils d'analyse (Grenon et Viau, 2007). De plus, ce type d'approche a permis d'étudier un groupe d'individus afin de décrire, comparer, répertorier, analyser et interpréter les éléments afférents à cette recherche (Cohen, Manion et Morrisson, 2008). Les techniques d'enquête ont été utiles pour recueillir des informations factuelles et des données sur les perceptions des enseignants quant à l'utilité des activités dans leur enseignement au quotidien ainsi que sur les activités de développement professionnel qu'ils privilégient (Weisberg, Krosnick et Bowen, 1996). Cependant, certaines limites sont liées à ce type d'enquête qui est de caractère ponctuel, dans la mesure où les résultats engendrés sont statiques, fixés dans un moment donné et qu'ils ne peuvent attester des modifications dans le temps.

\subsection{Déroulement}

Très tôt dans la démarche, il a été utile de préciser les concepts abordés de façon à les rendre observables et mesurables dans une perspective opératoire ainsi qu'à les rendre compréhensibles dans le contexte de la recherche. À partir de ces concepts, des indicateurs mesurables ont été isolés et sont devenus des variables (Howell, 1998; Simard, 2011). Ensuite, dans le processus de cette recherche, la population à l'étude a été ciblée et déterminée afin de maintenir une bonne représentativité. Puis, la collecte des données s'est faite à l'aide d'un questionnaire structuré autoadministré qui a été élaboré pour cette recherche. Il a été vérifié par des experts d'enquête, mis à l'essai par un prétest auprès de six enseignants volontaires et peaufiné, avant sa passation (Cohen, Manion et Morrison, 2008). La dernière étape a été l'analyse et l'interprétation des résultats, qui a consisté à comparer les données obtenues en lien avec les objectifs afin de faire ressortir les corrélations ou relations existantes (Caracelli et Greene, 1993). 


\subsection{Considérations éthiques}

Plusieurs précautions d'ordre éthique ont été prises pour cette recherche. Les répondants ont reçu toute l'information afin de leur permettre de faire un choix éclairé quant à leur participation. Ils se sont tous engagés de façon volontaire à répondre à un questionnaire en ligne, sans aucune possibilité de les identifier.

\subsection{Sujets}

La population à l'étude est constituée de 220 enseignants en exercice, ayant terminé leur formation initiale et exerçant au Saguenay-Lac-Saint-Jean, aux services éducatifs pour les jeunes. Le groupe de sujets est constitué de 167 femmes et 53 hommes dotés de 1 à 34 ans d'expérience, issus de plusieurs ordres d'enseignement (préscolaire, primaire et secondaire) et des 20 divers champs d'enseignement considérés par la convention collective de la Fédération des syndicats de l'enseignement (FSE, 2010). La sélection a été limitée aux enseignants de la région parce que celle-ci englobe quatre commissions scolaires, dans lesquelles on retrouve 98 écoles primaires et 19 écoles secondaires, et qu'elle embauche 3273 enseignants considérés à temps plein ou non. De plus, la répartition des enseignants de cette région est comparable à celle de l'ensemble du Québec, selon les statistiques de la Direction régionale du ministère de l'Éducation du Saguenay-Lac-Saint-Jean (2010) en ce qui concerne les variables utilisées.

\subsection{Instrumentation}

Aucun instrument de mesure en lien avec les objectifs de recherche n'a été répertorié dans les écrits consultés. Il a donc fallu en développer un spécifiquement pour cette recherche. Le questionnaire a été élaboré à partir d'un outil électronique, le logiciel d'enquête en ligne Lime Survey 1.91 +, ce qui a permis de compiler les données et de les transférer dans un logiciel d'analyse quantitative. Les participants ont eu 30 minutes pour répondre aux 48 questions du questionnaire transmis électroniquement. Celui-ci contient des questions précises qui ont été préalablement définies en fonction des compétences du référentiel de la formation en enseignement (ministère de l'Éducation du Québec, 2001) et des activités de développement professionnel, ainsi qu'une section d'information générale et sociodémographique. Ce type de questionnaire est largement utilisé pour la collecte de données d'enquête et il peut être administré sans la présence du chercheur (Blais et Durand, 2003; Cohen, et al. 2008; McLean, 2006).

\subsection{Méthodes d'analyse des données}

Les données ont été pondérées afin d'être représentatives de la population effective pour pallier certains écarts possibles. Un plan de dépouillement a été fait: une matrice a été créée avec le logiciel SPSS 17.0 au préalable, ce qui a permis de tester le questionnaire afin de vérifier s'il permettrait de répondre aux objectifs visés. 
Les données recueillies ont également été traitées à l'aide de ce logiciel. Les étapes de manipulation et d'analyse des données ont été réalisées à partir des perspectives d'analyse quantitatives d'Antonius (2003) et de Ouellet et Baillargeon (2004). Une analyse univariée a été faite à l'aide de mesures statistiques descriptives, telles la distribution de fréquences ainsi que les pourcentages qui ont constitué la mesure de tendance centrale utilisée dans cette recherche. Puis, l'analyse bivariée a été faite à l'aide du tableau de contingence pour permettre une analyse explicative entre les données obtenues. La statistique du khi-deux $\left(\chi^{2}\right)$ a été utilisée pour tester l'indépendance.

\section{Résultats}

Au total, 220 enseignants œuvrant aux services éducatifs pour les jeunes ont répondu au questionnaire de façon volontaire. Une proportion de $54 \%$ des répondants provient du primaire, $40 \%$ du secondaire et $6 \%$ du préscolaire. Cette répartition est la même que celle observée pour l'ensemble des enseignants du Saguenay-Lac-Saint-Jean (SLSJ), puisque la base de données a été pondérée selon cette caractéristique.

En ce qui concerne la fréquence des activités de développement professionnel privilégiées par les enseignants, l'analyse des données permet de constater que les activités pratiquées souvent et parfois par plus de $87 \%$ des répondants sont les suivantes: l'application de nouvelles techniques ou stratégies d'enseignement (DI), les formations offertes par des pairs ou des professionnels (DE), les lectures d'intérêt professionnel (DC), l'autocritique ou l'analyse des pratiques (DI) et la consultation d'ouvrages de référence (DE). Deux correspondent à la dimension interne (DI), deux à la dimension externe (DE) et une à la dimension combinée (DC).

Les données montrent également que certaines activités semblent moins obtenir la faveur des répondants. Plus de $75 \%$ d'entre eux disent ne jamais participer à certaines activités de formation telles que suivre des cours dans un établissement reconnu, participer à des recherches collaboratives ou à des actions menées par des chercheurs externes, participer à un groupe d'analyse des pratiques pédagogiques ou à un groupe de codéveloppement ou encore à une communauté professionnelle, participer à un programme de tutorat ou de mentorat ainsi que mener des recherches dans le cadre d'une maîtrise ou d'un doctorat. Ces cinq activités moins pratiquées par les enseignants appartiennent à la dimension combinée (DC).

De plus, les résultats montrent qu'il n'y aucune différence statistiquement significative selon le genre, l'ordre d'enseignement, les années d'expérience, pour plus de la moitié des activités de développement professionnel privilégiées par les enseignants. Le tableau 3 expose quelques différences statistiquement significatives observées selon le genre, et davantage pratiquées par les femmes. Quant au tableau 4, il indique celles que l'on observe selon l'ordre d'enseignement. 
Tableau 3

Activités privilégiées selon le genre

\begin{tabular}{lcccc}
\hline \multicolumn{1}{c}{ Activités } & \multicolumn{3}{c}{ Homme } & Femme \\
& Dimension & $\%$ & $\%$ & $X^{2}$ \\
\hline Nouvelles techniques ou stratégies d'enseignement & (DI) & 86,8 & 98,2 & $12,1^{* *}$ \\
Formations offertes par des pairs ou des professionnels & (DE) & 83,0 & 95,8 & $9,8^{* *}$ \\
Consultation de professionnels & (DE) & 73,6 & 86,2 & $4,6^{*}$ \\
Concertation entre collègues ou intervenants & (DC) & 60,4 & 79,2 & $7,5^{*}$ \\
\hline
\end{tabular}

${ }^{*} p<0,05 ;{ }^{*} p<0,01$

Tableau 4

Activités privilégiées selon l'ordre d'enseignement

\begin{tabular}{|c|c|c|c|c|c|}
\hline \multirow[b]{2}{*}{ Activités } & \multicolumn{4}{|c|}{ Ordre d'enseignement } & \multirow[b]{2}{*}{$x^{2}$} \\
\hline & & $\begin{array}{c}\text { Préscolaire } \\
\%\end{array}$ & $\begin{array}{c}\text { Primaire } \\
\%\end{array}$ & $\begin{array}{c}\text { Secondaire } \\
\%\end{array}$ & \\
\hline $\begin{array}{l}\text { Formations offertes par des pairs } \\
\text { ou des professionnels }\end{array}$ & $(D E)$ & 92,3 & 96,6 & 87,5 & $6,3^{*}$ \\
\hline Consultation de professionnels & $(D E)$ & 91,7 & 88,2 & 75,0 & $6,9^{*}$ \\
\hline Accompagnement de stagiaires & $(\mathrm{DC})$ & 83,3 & 65,5 & 46,6 & $10,6^{* *}$ \\
\hline Programme de tutorat ou de mentorat & $(\mathrm{DC})$ & 0,0 & 10,9 & 28,1 & $13,4^{* *}$ \\
\hline
\end{tabular}

${ }^{*} p<0,05 ;{ }^{* *} p<0,01$

D’autres résultats font ressortir des différences statistiquement significatives en ce qui concerne le nombre d'années d'expérience. Par exemple, les enseignants dans la phase de l'entrée ou du tâtonnement d'Huberman (1989), qui ont de 1 à 3 années d'expérience, mènent des recherches (DC) en plus grande proportion, tandis que peu d'enseignants de 4 à 6 ans d'expérience et aucun de 25 à 34 ans d'expérience ne disent en mener. En outre, les enseignants de 25 à 34 ans d'expérience, phase de la conservation, privilégient l'accompagnement de stagiaires en plus grand nombre.

Enfin, en ce qui concerne le champ d'enseignement, plus de la moitié des activités proposées dans le questionnaire montrent une différence statistiquement significative. Par exemple, la majorité des enseignants du primaire régulier, la totalité de ceux qui travaillent en adaptation scolaire et qui enseignent le français disent privilégier davantage l'application de nouvelles techniques. Puis, une plus grande proportion des enseignants du champ de l'adaptation scolaire, du français et du primaire régulier se distingue par la pratique de la concertation entre collègues.

On constate également que ces activités sont pratiquées dans le but de développer les compétences du référentiel de la formation en enseignement. Cependant, on remarque un écart entre le nombre moyen d'activités pratiquées et la compétence à développer. Le tableau 5 présente les trois compétences les plus développées en ordre décroissant. Par exemple, la compétence 4 est développée davantage avec une moyenne de cinq activités utilisées sur 15 proposées. 
Tableau 5

Compétences les plus développées par la pratique des activités en ordre décroissant

\begin{tabular}{|c|c|}
\hline Compétences & $\begin{array}{c}\text { Nombre moyen } \\
\text { d'activités pratiquées } \\
\text { dans le but de } \\
\text { développer la } \\
\text { compétence }\end{array}$ \\
\hline $\begin{array}{l}\text { Compétence } 4 \\
\text { Piloter des situations d'enseignement-apprentissage pour les contenus } \\
\text { à faire apprendre, et ce, en fonction des élèves concernés et du } \\
\text { développement des compétences visées dans le programme de formation }\end{array}$ & 5,0 \\
\hline $\begin{array}{l}\text { Compétence } 1 \\
\text { Agir en tant que professionnelle ou professionnel héritier, critique et } \\
\text { interprète d'objets de savoirs ou de culture dans l'exercice de ses fonctions }\end{array}$ & 4,6 \\
\hline $\begin{array}{l}\text { Compétence } 7 \\
\text { Adapter ses interventions aux besoins et aux caractéristiques des élèves } \\
\text { présentant des difficultés d'apprentissage, d'adaptation ou un handicap }\end{array}$ & 4,1 \\
\hline
\end{tabular}

Par contre, le tableau 6 indique les deux compétences les moins développées par les activités pratiquées. Entre autres, la compétence 2 avec une moyenne de 0,9 activités.

Tableau 6

Compétences les moins développées par la pratique des activités en ordre croissant

\begin{tabular}{lc}
\hline Compétences & $\begin{array}{c}\text { Nombre moyen } \\
\text { d'activités pratiquées } \\
\text { dans le but de } \\
\text { développer la } \\
\text { compétence }\end{array}$ \\
\hline $\begin{array}{l}\text { Compétence 2 } \\
\text { Communiquer clairement et correctement dans la langue } \\
\text { d'enseignement, à l'oral et à l'écrit, dans les divers contextes liés à la } \\
\text { profession enseignante }\end{array}$ & 0,9 \\
$\begin{array}{l}\text { Compétence } 12 \\
\text { Agir de façon éthique et responsable dans l'exercice de ses fonctions }\end{array}$ & 1,5 \\
\hline
\end{tabular}

En ce qui concerne l'utilité perçue des activités, les données indiquent que toutes les activités de développement professionnel pratiquées par les enseignants ont été jugées très utiles et assez utiles par plus de $79 \%$. Les activités perçues comme les plus utiles sont: la pratique de l'autocritique (DI), les recherches personnelles dans le cadre d'une maîtrise ou d'un doctorat (DC), la concertation entre collègues ou intervenants (DC), l'application de nouvelles techniques (DI) et la participation à des groupes d'analyse des pratiques (DC). Cependant, des enseignants ont dit trouver peu utiles certaines activités comme participer aux recherches actions ou aux recherches collaboratives menées par un chercheur externe (DC), assister à des 
colloques ou à des conférences (DE), faire des lectures sur des sujets d'intérêt professionnel (DC) et suivre des cours dans un établissement reconnu (DC).

Plus précisément, pour plusieurs activités, l'utilité ne varie pas selon les variables indépendantes du genre, de l'ordre, du champ d'enseignement et du nombre d'années d'expérience. Cependant, lors de l'analyse ciblée de la variable du genre, certaines activités semblent davantage jugées utiles par les enseignantes: la collaboration à des projets avec l'équipe enseignante, la concertation entre collègues, l'application de nouvelles techniques ou stratégies d'enseignement et la consultation de professionnels. De la même manière, en ce qui concerne la variable du champ d'enseignement, les résultats permettent de constater qu'il existe une différence significative pour certaines activités: les colloques, l'accompagnement de stagiaires et la concertation entre collègues sont perçues plus utiles par les enseignants des champs de l'adaptation scolaire et du préscolaire régulier. Quant à la variable du nombre d'années d'expérience, une activité est appréciée davantage des enseignants de 4 à 6 ans d'expérience (phase de la consolidation) et des enseignants de 7 à 24 ans d'expérience (phase de la diversification) : l'autocritique ou l'analyse des pratiques. Enfin, pour ce qui est de la variable de l'ordre d'enseignement, pour l'ensemble des activités, l'utilité ne varie pas.

\section{Discussion des résultats}

La collecte des données ainsi que l'analyse ont permis d'identifier les activités qui sont privilégiées pour le développement des compétences professionnelles selon les enseignants en exercice au préscolaire, au primaire et au secondaire, dans la région Saguenay-Lac-Saint-Jean. Ce portrait montre qu'une proportion importante d'enseignants s'investissent dans un processus de développement professionnel, et ce, nonobstant leur genre, leur ordre d'enseignement, leur champ d'enseignement et leur nombre d'années d'expérience. Les résultats appuient, dans une certaine mesure, les recherches recensées portant sur les types d'activités de développement professionnel pratiquées par les enseignants (Batal, 1997; Bélair, 2001; Glaser, 1987; Wittorski, 2000, 2004). Les résultats de cette recherche rappellent en effet différents travaux (Bellier, 1999; Van der Heijden et Barbier, 1999) qui ont mis en lien le développement des compétences et l'expérience comme source privilégiée pour acquérir le savoir pratique en enseignement, puisque l'enseignant, au cours de sa carrière, a la chance d'accumuler un ensemble complexe d'expériences pendant tout le processus du développement de ses compétences professionnelles (Holborn, 1992).

Les résultats font également ressortir le caractère réflexif lié au développement des compétences professionnelles, puisque celui-ci est intégré dans toutes les activités qui exigent la mobilisation des ressources internes et externes de la personne. Ainsi, la réflexion ne peut être écartée de la construction de la compétence. Ce point important rejoint les conclusions de nombreuses études (Altet, 2002; Guskey, 2000; Holborn, 1992; Schön, 1994; Stroobants, 1993). 
De plus, les résultats confirment qu'à des degrés variables, l'ensemble des douze compétences professionnelles du référentiel de la formation en enseignement (ministère de l'Éducation, du Loisir et du Sport 2001) sont mises à jour de façon continue par les enseignants (Defélix, Klarsfeld et Oiry, 2006; Fernagu-Oudet, 2001). Ces résultats corroborent ceux de Presseau et Frenay (2004), comme ceux de Girard et ses collaborateurs (1992), qui ont mentionné que l'enseignant doit posséder les compétences nécessaires pour gérer des activités complexes et que ces compétences professionnelles doivent être mises à jour en continu (Wittorski, 2007).

Cependant, les taux de fréquence observés des activités privilégiées sont différents, ce qui semble faire ressortir les affirmations de certains auteurs qui décrivent le développement des compétences professionnelles comme un parcours libre et individuel caractérisé par l'autonomie professionnelle des enseignants (Elliot, 1991; Gendron et Lafortune, 2009; Martineau et Gauthier, 2000).

Néanmoins, comme certaines activités sont encore peu pratiquées, plus précisément celles faisant appel à la dimension combinée, il nous semble que l'on peut se questionner sur les conditions à mettre en place pour faciliter la pratique de ces activités. Il s'agit là d'une autre avenue de recherche qui mérite d'être explorée. Les besoins et les conditions devraient être réévalués de manière à faciliter le développement professionnel des enseignants, compte tenu des effets bénéfiques du développement des compétences professionnelles sur la réussite de l'élève (Brassard, 2008; Bru, 2004). Cependant, au Québec, dans le contexte éducatif des dernières années, les enseignants ont été amenés à participer à des formations offertes par des pairs ou des professionnels concernant, en particulier, la réforme des programmes ou le renouvellement du curriculum. Ces formations, obligatoires également au Saguenay-Lac-Saint-Jean, peuvent peut-être expliquer, du moins en partie, la prédominance des activités des dimensions internes et externes sur celles de la dimension combinée. De plus, il ressort que les enseignants du préscolaire, en regard des autres ordres d'enseignement, privilégient l'accompagnement de stagiaires. Or, les étudiants au baccalauréat en enseignement au préscolaire et primaire de l'Université du Québec à Chicoutimi doivent tous faire un stage au préscolaire. Comme le nombre d'enseignants est moins élevé dans cet ordre d'enseignement, soit 189 sur 3273 enseignants au SaguenayLac-Saint-Jean, peut-être les enseignants ont-ils davantage l'occasion d'accompagner un stagiaire que les autres enseignants des ordres primaire et secondaire. Enfin, en région, des équipes d'enseignants du secondaire ont instauré des systèmes d'accompagnement de la relève et cet engagement est reconnu dans leur tâche globale. Un lien est à faire entre cette information et le fait que ces enseignants indiquent participer de manière plus fréquente que les enseignants des autres ordres d'enseignement à des programmes de tutorat ou de mentorat. Conséquemment, vu le contexte de la région, de telles hypothèses peuvent peutêtre expliquer en partie certains résultats obtenus. 


\section{Conclusion}

Cette recherche a permis d'établir un portrait comparatif des activités privilégiées pour le développement des compétences professionnelles chez les enseignants en exercice, en contextes préscolaire, primaire et secondaire au Saguenay-Lac-SaintJean. Cependant, elle ne peut permettre d'expliquer le choix qui a motivé le jugement des enseignants à l'égard des activités de développement professionnel. Les connaissances générées par cette enquête sont liées à des données présentées principalement sous forme de nombres, et l'analyse statistique a permis de dresser le portrait des activités privilégiées par les enseignants pour le développement de compétences professionnelles, sans toutefois expliquer ces préférences (Blalock, 1982; Boudreault, 2004; Colin, Lavoie, Delisle, Montreuil et Payette 1995; Howell, 1998; Krathwohl, 2009; Sowell, 2001; Trudel et Antonius, 1991).

Les résultats obtenus offrent un cadre de réflexion sur le renouvellement ou la bonification d'activités de développement professionnel en lien avec les compétences professionnelles, puisque le portrait est nuancé en fonction de diverses variables. De plus, ils permettent de comprendre l'utilité perçue des pratiques privilégiées du point de vue de 220 enseignants. Cependant, la reconnaissance de cette situation amène son lot de défis particuliers aux établissements quant aux conditions ou stratégies à favoriser en période de développement professionnel pour, à la fois, répondre aux besoins de développement professionnel des enseignants et promouvoir certains modes de formation ou compétences délaissées.

Un autre élément important ne peut être négligé. Il s'agit de la méthodologie employée avec les techniques d'enquête, car d'un point de vue praxéologique, elle permet l'utilisation d'un questionnaire qui répond à un besoin d'évaluation des activités de développement professionnel des enseignants. Cette méthodologie trouve une grande pertinence dans le fait que le système politique québécois fait de plus en plus référence à l'évaluation de la compétence des enseignants pour améliorer la performance des élèves et que des recherches prônent l'importance du développement professionnel pour améliorer les pratiques et satisfaire aux obligations de résultats (Normand et Derouet, 2011).

Enfin, les résultats soulèvent quelques questions: Comment expliquer le peu d'activités pratiquées en lien avec certaines compétences? Quelles pistes de solution peuvent être envisagées pour encourager la participation accrue à des activités de la dimension combinée? Voilà des questions qui méritent une attention particulière.

ENGLISH TITLE - Portrait of comparative activities of preferred professional development in preschool, primary and secondary contexts

SUMMARY - This article presents research that has developed a comparative portrait preferred by practicing teachers in preschool, primary and secondary contexts for the development of professional skills related to the profile repository teacher education activities in Quebec. The perceived usefulness of these activities was measured. This research used the survey technique and a questionnaire was developed. The results indicate that the proposed activities are used at varying frequencies according to the gender, number of years of experience, the order and 
the teaching field. However, the development of certain skills is neglected, which raises many questions. We expose the process and some results.

KEY WORDS • activities, skills, professional development.

TITULO - Retrato comparativo de las actividades de desarrollo profesional privilegiadas en la enseñanza en contextos de preescolar, primaria y secundaria

RESUMEN - En este artículo se presenta una investigación cuantitativa que ha desarrollado un retrato comparativo de las actividades privilegiadas por los profesores en ejercicio, en contextos de preescolar, primaria y secundaria, para el desarrollo de competencias profesionales relacionadas con el perfil de referencia de la formación del profesorado en Quebec. Se midió la percepción de la utilidad de estas actividades. Esta investigación utilizó la técnica de la encuesta y se elaboró un cuestionario. Los resultados indican que las actividades propuestas se usan con frecuencias variables según el sexo, el número de años de experiencia, el nivel escolar y el área de enseñanza. Sin embargo, el desarrollo de ciertas competencias es descuidado, lo que plantea numerosas preguntas. Exponemos el proceso y algunos resultados.

PALABRAS CLAVE • actividades, competencias, desarrollo profesional.

\section{Références}

Allal, L. (2002). Acquisition et évaluation des compétences en situation scolaire. Dans J. Dolz et E. Ollagnier (dir.), L'énigme de la compétence en éducation (p. 77-94). Bruxelles, Belgique: De Boeck.

Altet, M. (1996). Les dispositifs d'analyse des pratiques pédagogiques en formation d'enseignant: une démarche d'articulation pratique-théorie-pratique. Dans C. BlanchardLaVille et D. Fablet (dir.), L'analyse des pratiques professionnelles (p. 11-26). Paris, France: L'Harmattan.

Altet, M. (2001). Les compétences de l'enseignant-professionnel: entre savoirs, schèmes d'action et adaptation, le savoir analyser. Dans L. Paquay, M. Altet, É. Charlier et P. Perrenoud (dir.), Former des enseignants professionnels ( $3^{\mathrm{e}}$ édition) (p. 27-40). Bruxelles, Belgique: De Boeck.

Altet, M. (2002). Quelles professionnalités des formateurs en formation continue? Vers un profil polyidentitaire. Dans M. Altet, L. Paquay et P. Perrenoud (dir.), Formateurs d'enseignants. Quelle professionnalisation? (p. 59-87). Bruxelles, Belgique: De Boeck.

Altet, M. et Fabre M. (1994). Logiques et problématiques de l'articulation formation/ recherche dans les dispositifs de professionnalisation. Recherche et Formation, (17) 77-92.

Anadón, M. (1999). L’enseignement en voie de professionnalisation. Dans C. Gohier, N. Bednarz, L. Gaudreau, R. Pallascio et G. Parent (dir.), L'enseignant: un professionnel (p. 1-20). Québec, Québec: Presses de l’Université du Québec.

Anderson, L. W. (1986). La formation des maîtres en fonction des compétences attendues. Dans M. Crahay et D. Lafontaine (dir.), L'art et la science de l'enseignement (p. 365-385). Bruxelles, Belgique: Labor.

Antonius, R. (2003). Interpreting quantitative data with SPSS. London, United-Kingdom: SAGE publications.

Batal, C. (1997). La gestion des ressources humaines dans le secteur public. L'analyse des métiers, des emplois et des compétences. Paris, France: Édition d'Organisation. 
Becher, T. (1999). Professional practices: commitment and capability in a changing environment. New Brunswick, New Jersey: Transaction Publishers.

Bélair, L. (2001). La formation à la complexité du métier d'enseignant. Dans L. Paquay, M. Altet, É. Charlier, et P. Perrenoud (dir.), Former des enseignants professionnels (3e édition) (p. 63-75). Bruxelles, Belgique: De Boeck.

Bellier, S. (1999). La compétence. Dans P. Carré et P. Caspar (dir.), Traité des sciences et des techniques de la formation (p. 223-244). Paris, France: Dunod Éditeur.

Blais, A. et Durand, C. (2003). Le sondage. Dans B. Gauthier (dir.), Recherche sociale: de la problématique à la collecte des données (3e édition révisée) (p. 387-429). Québec, Québec: Presses de l'Université du Québec.

Blalock, H. (1982). An introduction to social research (2 ${ }^{\text {nd }}$ edition). Englewood Cliffs, New Jersey: Prentice-Hall.

Boudreault, P. (2004). La recherche quantitative. Dans T. Karsenti et L. Savoie-Zajc (dir.), La recherche en éducation: étapes et approches (p. 150-180). Sherbrooke, Québec: Édition du CRP.

Bouteiller, D. (2009). Le syndrome du crocodile et le défi de l'apprentissage continu. Dans D. Bouteiller, et L. Morin (dir.), Développer les compétences au travail (p. 199-223). Montréal, Québec: HEC.

Brassard, A. (2008). Référentiel de compétences et élaboration de programmes en gestion de l'éducation: «c'est un peu plus compliqué que ça». Dans M. Ettayebi, R. Opertti et P. Jonnaert (dir.), Logique de compétences et développement curriculaire. Débats, perspectives et alternative pour les systèmes éducatifs (p. 237-252). Paris, France: L'Harmattan.

Bru, M. (2004). Quelles compétences pour enseigner? Dans V. Hajjar, et A. Baubion-Broye (dir.), Modèles et méthodologies d'analyse des compétences (p. 40-50). Toulouse, France: Octarès Éditions.

Buhot, E. et Cosnefroy, L. (2011). De la formation initiale à la première affectation, comment les professeurs des écoles perçoivent-ils leur développement professionnel? Les sciences de l'éducation - Pour l'ère nouvelle, 44(1), 17-35.

Campbell, D. M., Cignetti, P. B., Melenyzer, B. J., Nettles, D. H. and R. M. J. Wyman (1997). How to develop a professional portfolio. Boston, Massachusetts: Allyn and Bacon.

Caracelli, V. J. and Greene, J. C. (1993). Data analysis strategies for mixed-method evaluation designs. Educational evaluation and policy analysis, 15(2), 195-207.

Carnus, M. F., Garcia-Debanc, C. et Terrisse, A. (dir.). (2008). Analyse des pratiques des enseignants débutants: approches didactique. Grenoble, France: La pensée sauvage.

Clément, M. and Vandenberghe, R. (2000). Teachers' professional development: a solitary or collegial adventure? Teaching and teacher education, 16(1), 81-101.

Cochran-Smith, M. and Lytle, S. (1999). The teacher research movement: a decade later. Educational research, 28(6), 15-25.

Cohen, L., Manion, L. and Morrison, K. (2008). Research methods in education ( $6^{\text {th }}$ edition). London, United Kingdom: Routledge.

Colin, M., Lavoie, P., Delisle, M., Montreuil, C. et Payette, C. (1995) Initiation aux méthodes quantitatives en sciences humaines (2 édition). Boucherville, Québec: Gaëtan Morin éditeur. 
Courcy, É. (2002). La triade. Points de vue de stagiaires. Dans M. Boutet et N. Rousseau (dir.), Les enjeux de la supervision pédagogique des stages (p. 97-103). Québec, Québec: Presses de l'Université du Québec.

Craft, A. (2000). Continuing professional development ( $2^{\text {nd }}$ edition). London, UnitedKingdom: Falmer.

Daele, A. et Charlier B. (2004). Comprendre les communautés de pratiques virtuelles d'enseignants: pratiques et recherches. Paris, France: L'Harmattan.

Day, C. (1999). Developing teachers: the challenges of lifelong learning. London, UnitedKingdom: Falmer.

De Bock, J. (2000). De la formation du personnel au développement des compétences. Dans C. Bosman, F. M. Gérard et X. Roegiers (dir), Quel avenir pour les compétences? (p. 51-57). Bruxelles, Belgique: De Boeck.

Defélix, C., Klarsfeld, A. et Oiry, E. (dir). (2006). Nouveaux regards sur la gestion des compétences. Paris, France: Éditions Vuibert.

Direction régionale du ministère de l'Éducation du Saguenay-Lac-St-Jean (2010). Statistiques sur le personnel de l'ensemble des commissions scolaires 2009-2010. Québec, Québec: ministère de l'Éducation du Québec.

Durand, M. (1996). L'enseignement en milieu scolaire. Paris, France: Presses universitaires de France.

Elliot, J. (1991). A model of professionalism and its implications for teacher education. British educational research journal, 17(4), 309-318.

Fédération des syndicats de l'enseignement (2010). Convention collective nationale. Loi sur le régime de négociation des conventions collectives dans les secteurs public et parapublic. Québec, Québec: Fédération des syndicats de l'enseignement.

Fernagu-Oudet, S. (2001). Voyage au cœur de la compétence ou pour une épistémologie de la pratique. Actualité de la formation permanente, (170), 8-20.

Flück, C. (2001). Compétences et performances: une alliance réussie. Paris, France: Demos.

Fourez, G. (1999). Compétences, contenus, capacités et autres casse-tête. Forum pédagogies, (16), 26-31.

Gauthier, C. et Mellouki, M. (2003). Attirer, former et retenir des enseignants de qualité au Québec. Rapport présenté à l'OCDE. Québec, Québec: ministère de l'Éducation du Québec.

Gendron, B. et Lafortune, L. (dir.). (2009). Leadership et compétences émotionnelles: dans l'accompagnement au changement. Québec, Québec: Presses de l'Université du Québec.

Girard, L., McLean, E. et Morissette, D. (1992). Supervision pédagogique et réussite scolaire. Boucherville, Québec: Gaëtan Morin éditeur.

Glaser, B. G. (1987). Theoretical sensitivity. Advances in the methodology of grounded theory. Mill Valley, California: Sociology Press.

Gohier, C., Anadón, M., Bouchard, Y., Charbonneau, B. et Chevrier, J. (1999). Vers une vision renouvelée de la professionnalisation de l'enseignement et de la construction de l'identité professionnelle de l'enseignant. Dans C. Gohier, N. Bednarz, L. Gaudreau, R. Pallascio et G. Parent (dir.), L'enseignant un professionnel (p. 21-56). Québec, Québec: Presses de l'Université du Québec. 
Grenon, G. et Viau, S. (2007). Méthodes quantitatives en sciences humaines. Volume 1. Montréal, Québec: Chenelière Éducation.

Guillemette, F. (2006). L'engagement des enseignants du primaire et du secondaire dans leur développement professionnel (thèse de doctorat non publiée). Montréal, Québec: Université du Québec à Montréal, Université du Québec à Trois-Rivières.

Guskey, T. R. (2000). Evaluating professional development. Thousand Oaks, California: Corwin Press.

Hargreaves, A. (1995). Beyond collaboration: critical teacher development in the postmodern age. Dans J. Smyth (dir.), Critical discourses on teacher development (p. 149179). London, United-Kingdom: OISE.

Hendricson, W. D. and Kleffner, J. H. (1998). Curricular and instructional implications of competency-based dental education. Journal of dental education, 62(2), 183-196.

Holborn, P. (1992). Devenir un praticien réflexif. Dans P. Holborn, M. Wideen et I. Andrews (dir.), Devenir enseignant. D’une expérience de survie à la maîtrise d'une pratique professionnelle (p.85-103). Montréal, Québec: Les Éditions Logiques.

Holh, J. (1993). L'enseignant des années 90, un professionnel en transition. Dimensions, (11), 10-11.

Howell, D. C. (1998). Méthodes statistiques en sciences humaines. Bruxelles, Belgique: De Boeck.

Huberman, M. (1989). Les phases de la carrière enseignante. Revue française de pédagogie, (86), 5-16.

Jonnaert, P. (2002). Compétences et socioconstructivisme. Un cadre théorique. Bruxelles, Belgique: De Boeck.

Kalika, M. (1998). Quelles conditions les systèmes d'accréditation doivent-ils remplir? Dans Institut de la Méditerranée (dir.), L’accréditation des compétences dans la société cognitive. Actes de conférence (p. 117-129). Marseille, France: Éditions de l’Aube.

Krathwohl, D. (2009). Methods of educational and social science research. The logic of methods ( $3^{\text {rd }}$ edition). Long Grove, Illinois: Waveland Press.

Lafortune, L., Deaudelin, C. et Deslandes, R. (2001). Formation à l'accompagnement dans une optique réflexive et métacognitive. Dans L. Lafortune, C. Deaudelin, P.-A. Doudin, et D. Martin (dir.), La formation continue. De la réflexion à l'action (p. 45-71). Québec, Québec: Presses de l'Université du Québec.

Learned, W. S. and Bagley, W.C. (1988). The professional preparation of teachers for american public schools. New York, New York: Carnegie foundation for the advancement of teaching.

Le Boterf, G. (2001). Construire les compétences individuelles et collectives (2 édition). Paris, France: Édition d'Organisation.

Le Boterf, G. (2008). Repenser la compétence. Pour dépasser les idées reçues: quinze propositions. Paris, France: Eyrolles.

Lebuis, P., Bednarz, N. et Desgagné, S. (1995). Recherche collaborative et formation continue: un nouveau rapport entre recherche et pratique professionnelle. Changement sociétal et recherche en éducation. Actes du colloque-réseau de doctorat en éducation tenu à l'Université du Québec à Chicoutimi (p. 173-190). Chicoutimi, Québec: ÈRE-2000. 
Leclerc, M. (2012). Communauté d'apprentissage professionnelle. Guide à l'intention des leaders scolaires. Québec, Québec: Presses de l’Université du Québec.

Legendre, R. (2005). Dictionnaire actuel de l'éducation. Montréal, Québec: Guérin éditeur.

Lesourne, J. (1988). Éducation et société. Les défis de l'an 2000. Paris, France: Éditions La Découverte/Le monde de l'éducation.

Lévy-Leboyer, C. (1996). La gestion des compétences. Paris, France: Éditions d’Organisation.

Lieberman, A. et McLaughlin, M. W. (2000). Le perfectionnement professionnel aux États-Unis: principes et réalités. Perspectives, 30(2), 253-267.

Martineau, S. (2006). La question des compétences: tour d'horizon socio-historique de la notion et analyse conceptuelle. Université du Québec à Trois-Rivières, Québec: Laboratoire d'analyse de l'insertion professionnelle en enseignement (LADIPE).

Martineau, S. et Gauthier, C. (2000). La place des savoirs dans la construction de l'identité professionnelle collective des enseignants ou le paradoxe de la qualification contre la compétence. Dans C. Gohier, et C. Alin (dir.), Enseignant-Formateur: la construction de l'identité professionnelle (p. 85-110). Paris, France: L'Harmattan.

Massot, P. et Feisthammel, D. (1997). Conduites professionnelles, conduites de management. Un outil de développement des compétences. Rueil-Malmaison, France: Éditions Liaisons.

McLean, J. E. (2006). From quantitative to qualitative and half way back. Foreword, 13(1), 3-4.

Ministère de l'Éducation du Québec (1999). Choisir plutôt que subir le changement. Orientations pour la formation continue du personnel enseignant. Québec, Québec: Ministère de l'Éducation du Québec.

Ministère de l'Éducation du Québec (2001). Programme de formation à l'enseignement: les orientations et les compétences professionnelles. Québec, Québec: Bibliothèque nationale du Québec.

Mukamurera, J. Bourque, J. et Gingras, C. (2008). Portraits et défis de l'insertion dans l'enseignement au Québec pour les nouvelles générations d'enseignants. Dans L. Portelance, J. Mukamurera, S. Martineau et C. Gervais (dir.), Insertion dans le milieu scolaire. Une phase cruciale du développement professionnel de l'enseignant (p. 49-72). Québec, Québec: Les Presses de l'Université Laval.

Normand, R. et Derouet, J.-L. (2011). Évaluation, développement professionnel et organisation. Revue française de pédagogie, 1(174), 5-20.

Orange, C. (2006). Analyse de pratiques et formation des enseignants. Un point de vue didactique. Recherche et formation, (51)119-131.

Organisation de coopération et de développement économiques (1998). L'école à la page. Formation continue et perfectionnement professionnel des enseignants. Paris, France: Organisation de coopération et de développement économiques.

Ouellet, F. et Baillargeon, G. (2004). Analyse de données avec SPSS pour Windows versions 10.0 et 11.0. Montréal, Québec: Les Éditions SMG.

Paquay, L. (2000a). L'évaluation, couperet ou levier du développement des compétences professionnelles? Dans C. Bosman, F.-M. Gerard, et X. Roegiers (dir.), Quel avenir pour les compétences? (p. 119-134). Bruxelles, Belgique: De Boeck. 
Paquay, L. (2000b). Quand un stage vise la diffusion de dispositifs innovants et la professionnalisation des enseignants. Dans G. Carlier, J.-P. Renard et L. Paquay (dir.), La formation continue des enseignants. Enjeux, innovation et réflexivité (p. 155-182). Bruxelles, Belgique: De Boeck.

Paquette, G. (2002). Modélisation des connaissances et des compétences. Québec, Québec: Presses de l'Université du Québec.

Parent, G., Corriveau, L., Savoie-Zajc, L., Dolbec, A., Cartier, R., Toussaint, P., Laurin, P. et Bonneau, G. A. (1999). Formation continue du personnel enseignant: vers une culture de développement professionnel. Dans C. Gohier, N. Bednarz, L. Gaudreau, R. Pallascio et G. Parent (dir.), L'enseignant: un professionnel (p. 119-143). Québec, Québec: Presses de l'Université du Québec.

Perrenoud, P. (1999). Gestion de l'imprévu, analyse de l'action et construction de compétences. Éducation permanente, (140), 123-144.

Perrenoud, P. (2001). Fondements de l'éducation scolaire: enjeux de socialisation et de formation. Dans C. Gohier et S. Laurin (dir.), Entre culture, compétence et contenu: la formation fondamentale, un espace à redéfinir (p. 55-84). Montréal: Les Éditions Logiques.

Pinte, G. (2004). La validation des acquis de l'expérience: un développement des compétences par l'autoformation qui redonne du sens au concept d'éducation permanente. Dans R. Toussaint et C. Xypa (dir.), La notion de compétence en éducation et en formation (p. 207-225). Paris, France: L'Harmattan.

Portelance, L., Mukamurera, J., Martineau, S. et Gervais, C. (dir.) (2008). Insertion dans le milieu scolaire. Une phase cruciale du développement professionnel de l'enseignant (p. 49-72). Québec, Québec: Les Presses de l'Université Laval.

Presseau, A. et Frenay, M. (2004). Le transfert des apprentissages. Comprendre pour mieux intervenir. Québec, Québec: Les Presses de l'Université Laval.

Presseau, A., Martineau, S. et Miron, J.-M. (2002). Vers un dispositif de formation des enseignants favorable à la réflexion et au développement de compétences transférables. Dans M. Boutet et N. Rousseau (dir.), Les enjeux de la supervision pédagogique des stages (p. 97-103). Québec, Québec: Presses de l’Université du Québec.

Prud'homme, L. (2007). La différenciation pédagogique: analyse du sens construit par des enseignantes et un chercheur-formateur dans un contexte de recherche-action-formation (thèse de doctorat non publiée). Gatineau, Québec: Université du Québec en Outaouais.

Saint-Pierre, L. et Lison, C. (2012). Prise en compte de composantes identitaires du personnel enseignant du collégial dans la formation continue. Dans L. Saint-Pierre (dir.), La formation continue et l'accompagnement du personnel enseignant du collégial (p. 25-49). Montréal, Québec: Chenelière Éducation.

Savoie-Zajc, L. et Dionne, L. (2001). Vers la mise en place d'une culture de formation continue dans les milieux scolaires. Dans L. Lafortune, C. Deaudelin, P.-A. Doudin et D. Martin (dir.), La formation continue. De la réflexion à l'action (p. 139-164). Québec, Québec: Presses de l'Université du Québec.

Schön, D. A. (1994). Le praticien réflexif. Montréal, Québec: Les Éditions Logiques.

Simard, C. (2011). Méthodes quantitatives: approche pédagogique progressive en sciences humaines (4 édition.). Montréal, Québec: Modulo.

Sowell, E. J. (2001). Design procedures for experimental research. Educational research: an 
interactive introduction. Boston, Massachusetts: McGraw-Hill.

Stroobants, M. (1993). Savoir-faire et compétences au travail: une sociologie de la fabrication des aptitudes. Bruxelles, Belgique: Éditions de l’Université de Bruxelles.

Tardif, J. (2006). L'évaluation des compétences: documenter le parcours de développement. Montréal, Québec: Chenelière Éducation.

Théberge, M., Bourrassa, M., Lauzon, Y. et Huard-Wart, G. (1997). Vers un modèle de cohérence entre formation et pratique et formation théorique. Revue des sciences de l'éducation, 23(2), 345-370.

Toupin, L. (1995). De la formation au métier: savoir transférer ses connaissances dans l'action. Paris, France: ESF éditeur.

Trudel, R. et Antonius, R. (1991). Méthodes quantitatives appliqués aux sciences humaines. Montréal, Québec: Centre éducatif et culturel-CEC.

Van Der Heijden, B. et Barbier, G. (1999). La compétence, sa nature et son développement. Éducation permanente, (140), 119-137.

Vonk, J.C. (1988). L'évolution professionnelle des enseignants débutants et ses répercussions sur la formation initiale et continue. Recherche et formation, 3(3), 47-60.

Weisberg, H. F., Krosnick, J. A. and Bowen, B. D. (1996). An introduction to survey research, polling, and data analysis ( ${ }^{\text {rd }}$ edition.). Thousand Oaks, California: SAGE Publications.

Wittorski, R. (2000). La production de compétences collectives par et dans l'analyse des pratiques professionnelles. Psychologie du travail et des organisations, 6(3-4), 75-102.

Wittorski, R. (2004). Savoirs d'action, compétences et professionnalisation. Dans J. P. Astolfi (dir.), Savoirs en action et acteurs de la formation (p. 51-76). Rouen, France: Presses universitaires de Rouen.

Wittorski, R. (2007). Professionnalisation et développement professionnel. Paris, France: L'Harmattan.

\section{Correspondance}

nathalie.murray@cjonquiere.qc.ca

\section{Contribution de l'auteure}

Nathalie Murray: $100 \%$

Ce texte a été révisé par Caroline de Launay

Texte reçu le: 28 novembre 2013

Version finale reçue le: 19 août 2014

Accepté le: 19 août 2014 